\title{
Molecular study on extended spectrum $\beta$-lactamase- producing Gram negative bacteria isolated from Ahmadi Hospital in Kuwait
}

\author{
Eshaq A. Mohmid ${ }^{1 *}$, El-Sayed A. El-Sayed ${ }^{2}$ and Mahmoud F. Abdel El-Haliem ${ }^{2}$ \\ ${ }^{1}$ Department of Microbiology, Medical Laboratory, Ahmadi Hospital, Kuwait Oil Company, Ahmadi, Kuwait. \\ ${ }^{2}$ Department of Botany, Faculty of Science, Zagazig University, Zagzig, Egypt.
}

Accepted 26 July, 2013

\begin{abstract}
During the period from November 2009 to April 2010, 84 out of 560 extended spectrum $\beta$-lactamase (ESBL) producing negative bacteria were isolated from patients in different departments of the Ahmadi Hospital in Kuwait. The isolates were collected from urine catheter, wound, sputum, blood and other different samples. The ESBL infection rate in the in-patients was $62 \%$ and part of them (19\%) were in the intensive care unit. All the isolated bacteria were identified and tested for antimicrobial susceptibility using an automated system (VITEK 2) and different antibiotic discs (15) by standard disc diffusion. The number of the recorded isolated multi-resistant Gram's negative bacteria was 54 isolates of Escherichia coli, 18 of Klebsiella pneumoniae, 11 of Pseudomonas aeruginosa, six of Proteus mirabilis, five of Enterobacter cloacae, four of Acinetobacter baumanii and one of Enterobacter aerogenes. They were resistant to the third generation of cephalosporins; Ceftazidime, Cefotaxime and Ceftriaxone. Meropenam (MEM) was the highest effective antibiotic against all the isolated bacteria (86\%). The production of the ESBL was detected by phenotypic methods using E-test (96.4\%), double disk synergy test (95\%) and VITEK $2(84.5 \%)$ in all multi-resistant isolates except $A$. baumanii and $P$. aeruginosa. All ESBL producing isolates were extracted and subjected to PCR using blaSHV, blaCTX-M and blaTEM primers. The bla-CTX-M (63.1\%) was the most predominant ESBL gene that was produced in abundance by 42 isolates of $E$. coli. The most predominant ESBL isolates producing bla-TEM, blaCTX-M and bla-SHV genes were successfully identified by $16 \mathrm{~S}$ rDNA. The conjugation assay between $E$. coli HB101 and the most predominant ESBL producing E. coli showed that the bla-CTX-M gene was able to be transferrable suggesting that they were plasmid mediated.
\end{abstract}

Key words: Extended spectrum $\beta$-lactamase (ESBL), VITEK 2, E-Test, DDST, polymeric chain reaction (PCR), 16S rDNA.

\section{INTRODUCTION}

Extended spectrum of $\beta$-lactamases (ESBLs) are enzymes produced by a variety of Gram negative bacteria which confer an increased resistance to commonly used antibiotics. They are a worrying global public health issue as infections caused by such enzyme producing organisms are associated with a higher morbi- 
dity and mortality with greater fiscal burden. Coupled with increasing prevalence rates worldwide and an ever diminishing supply in the antibiotic armamentarium, these enzymes represent a clear and present danger to public health (Dhillon and Clark, 2011). The introduction of the third-generation of cephalosporins into clinical practice in the early 1980s is heralded as a major breakthrough in the fight against $\beta$-lactamase-mediated bacterial resistance to antibiotics.

These cephalosporins have been developed in response to the increased prevalence of B-lactamases in certain organisms (for example, ampicillin hydrolyzing TEM-1 and SHV-1 $\beta$-lactamases in $E$. coli and $K$. pneumoniae). The third generation of cepha-losporins against most $\beta$-lactamase-producing organisms has major advantages of lessened nephrotoxic effects compared to aminoglycosides and polymyxins. The first report of plasmid-encoded $\beta$-lactamases capable of hydrolyzing the extended-spectrum cephalosporins, SHV2 , was published from a strain of $K$. ozaenae (Knothe et al., 1983). Among Enterobacteriaceae, extended spectrum beta lactamases (ESBLs) have been found mainly in Klebsiella spp. and E. coli, but have been also reported in another genera, such as Citrobacter, Enterobacter, Morganella, Proteus, Providencia, Salmonella, Serratia and Pseudomonas spp. (Arlet and Philippon, 1991; Ivanova et al., 2008). Thereafter, the number of ESBL variants occurring through amino acid mutations has progressively increased while demonstrating geographic variations (Winokur et al., 2001).

SHV-types of ESBLs are mostly derivatives of a nonESBL SHV-1 and quickly invaded several continents (Bradford, 2001; Paterson et al., 2003). The majority of plasmid-mediated beta-lactamases, namely, TEM-1 or less frequently, TEM-2 are broad-spectrum betalactamases which do not hydrolyze oxyimino-cephalosporins or aztreonam. The (CTX-M) family, first described in 1992, is known to be the most dominant non-TEM, nonSHV ESBL among Enterbacteriaceae and it was recognized as a rapidly growing family of ESBLs that prefer to hydrolyze cefotaxime rather than ceftazidime (Bauernfeind et al., 1992; Bonnet, 2004). Several researchers used genotypic methods for the identification of the specific gene responsible for the production of the ESBLs, which have the additional ability to detect lowlevel resistance; that is, it can be missed by phenotypic methods (Woodford and Sundsfjord, 2005). Furthermore, molecular assays also have the potential to be done directly on clinical specimens without culturing the bacteria, with subsequent reduction of detection time (Tenover, 2007). Olsen and Woese (1993) showed that the $16 \mathrm{~S}$ ribosomal ribonucleic acid (16S rRNA) gene, since the discovery of polymeric chain reaction (PCR) and deoxyribonucleic acid (DNA), was highly conserved within and among species of the same genus in compar- ring the gene sequences of the bacterial species. Hence, it can be used as the new gold standard for identification of bacteria to the species level of bacterial strains that have posed problems for the accurate identification of such isolates.

The genes that encode ESBLs were frequently found on the same plasmids as genes that encode resistance to aminoglycosides and sulfonamides. Many bacterial species possess changes that confer high-level resistance to quinolones. As a result, ESBL-producing bacterial species in hospitals and intensive care units (ICU) are commonly multidrug resistant, which possess a particular challenge for the treatment of nosocomial infections, especially in critically ill patients. Inappropriate empiric antimicrobial treatment for nosocomial- or community- acquired infections has been reported to contribute to significantly greater mortality rates in the $\mathrm{ICU}$, and inadequate antimicrobial treatment of infection was the most important independent determinant of hospital mortality (Kollef et al., 1999). ESBLs are increasingly spreading among Enterobacteriaceae (clinical isolates) throughout the world due mostly to their presence on highly conjugative plasmid. Surveys that are done in Canada, Greece, United Kingdom and Italy showed an association between the CTX-M type of ESBL and resistance to other antimicrobial agents (Bonnet, 2004). This is explained by a number of findings showing that bla-CTX-M genes are commonly found on large plasmids that often carry other genes conferring resistance to other antimicrobial agents including aminoglycosides, fluoroquinolones, chloramphenicols, tetracyclins and others; particularly, bla-OXA-1, bla-TEM-1 (Leflon-Guibout et al., 2004; Bratu et al., 2005).

The aim of this work was to determine the prevalence of ESBL producing members of the Gram negative isolates which are isolated from samples collected from different departments at the Ahmadi Hospital in Kuwait by means of phenotypic and genotypic methods and also, characterize the genetic basis of ESBL producing isolates and compare them with the universal isolates of the gene bank.

\section{MATERIALS AND METHODS}

\section{Bacterial isolates and sample collection}

Totally, 560 clinical significant bacterial strains belonging to the family Enterobacteriaceae were isolated from patients seen and treated at the Ahmadi Hospital in Kuwait from November 2009 to April 2010. Isolates found to be resistant or with decreased susceptibility to any of the third generation of cephalosporins such as ceftazidime, cefotaxime or ceftriaxone were selected for ESBL testing (Duttaroy and Mehta, 2005; Paterson and Bonomo, 2005). The samples were collected from different departments and sent to the microbiology laboratory. They were (465) urine, (17) sputum, (23) catheter, (11) blood, (20) wound and (24) different samples. 
The selected isolates were reviewed and recorded for the work of the study; including patient file number, diagnosis, age, sex, type of sample, location and type of infected organism.

\section{Identification of gram negative bacteria}

The samples were cultured on blood and MacConkey agar media (OXOID). The plates were incubated at $37^{\circ} \mathrm{C}$ for $24 \mathrm{~h}$, and the Gram negative bacterial colonies were selected for identification and antimicrobial susceptibility testing by VITEK 2 (BioMerieux, Marcy L'Etoile, France) using ID-GNI and AST-GN27 cards according to the manufacturer's instruction (NCCLS, 1999). Then, the results were interpreted by using software version VTK2-R 4.01, an advanced expert system (AES) (Livermore, 1995; Canton et al., 2001; Sanders et al., 2001). E. coli (ATCC 25922) and $P$. aeruginosa (ATCC 27853) were used for negative quality control processing in all experimental tests as recommended by clinical and laboratory standards institute (CLSI, 2005).

\section{Criterion for selection of ESBL producing strains}

Routine disc diffusion susceptibility test of the isolates was performed by standard disk diffusion; Bauer-Kirby method (Bauer et al., 1966). All the isolates with the control strains were inoculated according to McFarland standards (McFarland, 1907; Farmer et al., 2007) and streaked on Mueller Hinton agar medium (Oxoid Ltd., Basingstoke, UK) using sterile cotton swabs. Then, 15 different antibiotic discs (MAST GROUP Ltd, UK), belonging to 8 groups of $\beta$ - and non $\beta$-lactam agents, -were added onto the plates and incubated at $37^{\circ} \mathrm{C}$ for $24 \mathrm{~h}$. The activity of each antibiotic disc was determined by measuring the antibiotic zone diameter (NCCLS, 2003). Following the CLSI criteria; any resistant with one of the third generation cephalosporins is selected for ESBL detection and confirmation (CLSI, 2005).

\section{ESBL phenotypic tests}

All the isolated resistant gram negative bacteria that are resistant to the third generation cephalosporins were tested by VITEK 2, E-Test (AB Biodisk, Sweden) and double disc synergy test (DDST) methods. VITEK2 is based on the detection of the inhibitory effect of clavulanic acid on ESBLs in the presence of either cefotaxime or ceftazidime. E-test strips of cefotaxime/cefotaxime + clavulanic acid (CT/ CTL) and ceftazidime/ceftazidime + clavulanic acid (TZ/TZL) are designed to confirm the presence of clavulanic acid that is able to inhibit ESBL enzymes in E. coli, K. pneumoniae, K. oxytoca and other relevant species (Cormican et al., 1996).

DDST is used to reconfirm the strains that are ESBL positive tested by E- test and VITEK 2 and employed three discs, ceftazidime $(30 \mu \mathrm{g})$, cefotaxime $(30 \mu \mathrm{g})$ and amoxicillin with clavulanic acid $(30 \mu \mathrm{g})$ from Oxoid. The discs were placed with their centers at the recommended distance $(25$ to $30 \mathrm{~mm})$ apart from each other on the plat. When the inhibition zone of ceftazidime or cefotaxime in combination singly with clavulanic acid is enhanced (zone diameter $\geq 5$ ), it confirms an ESBL producing organism as recommended by the NCCLS (2000).

\section{ESBL molecular tests}

The ESBL isolates were tested in vitro as follows:

\section{Extraction of ESBLS}

One loopful of overnight growth ESBL isolates with the positive control strains was inoculated into $1.0 \mathrm{ml}$ sterile distilled water. The bacterial suspension was emulsified and boiled for $10 \mathrm{~min}$ at $100^{\circ} \mathrm{C}$ and then kept for 5 min on ice. The emulsified suspension was centrifuged for $10 \mathrm{~min}$ at $12000 \mathrm{rpm}$ and 300 to $400 \mathrm{ul}$ from the supernatant was stored at $-20^{\circ} \mathrm{C}$ (Lou et al., 1993; Merk et al., 2006).

\section{Amplification and sequencing of ESBL genes}

PCR analysis in vitro was performed on all isolates with the positive control strains containing specific primers to confirm the presence of ESBL. The ESBL coding regions were amplified using the primers listed in Table 1 and a cell suspension as template containing 2.5 ul buffer, $2.0 \mathrm{ul} \mathrm{MgCl}^{2}$ (Promega, Ltd, UK), $0.5 \mathrm{ul}$ dNTP, 0.25 ul Taq DNA polymerase (Promega, Ltd, UK), $1.0 \mathrm{ul}$ forward and retained primers, 2.0 ul DMSO (only for blaSHV primer), $2.0 \mathrm{ul}$ extracted DNA and sterile water for the remaining 25.0 ul (Dashti et al., 2009). The PCR products were analyzed by agarose gel electrophoresis using $1.5 \%$ (wt/vol) in a programmable PCR master cycler gradient (Eppendorf, Germany) following the technique described by Tenover et al. (1995) and photographed using a gel documentation system (UVP Company, Upland, CA, USA). E. coli strain ATCC 25922 was used as a negative control in all PCR assays. The positive control for SHV was K. pneumoniae 6064, whilst the positive control for TEM and CTX-M was E. coli 971 (Dashti et al., 2010). The blaTEM and blaCTX-M PCR products were purified using the NucleoSpin Extract II (Macherey-Nagel, Duren, Germany) and sequenced using the AB13100 DNA sequencing system (Applied Biosystem, Foster City, CA, USA). The resulting DNA sequences were compared with ESBL producing gram negative bacteria in the gene bank using BLAST at the website (http:// www.ncbi.nih.gov/blas).

\section{Identification by 16SrDNA}

Total DNA was extracted from the most predominant ESBL producing isolates and amplified using 16SrRNA primers listed in Table 1. Presence and yield of specific PCR products (16S rRNA gene) were monitored by running $1 \%$ agarose gels and cleaned up by using GeneJET TM PCR purification kit (Fermentas). The amplified DNA fragments were sequenced at GATC Biotech AG (Konstanz, Germany) using ABI 3730xI DNA sequencing system and the 16S rDNA sequences were analyzed by using the basic local alignment search tool (BLAST) program (http://www.ncbi.nlm.nih.gov/blast).

\section{Conjugation assay}

The recipient cell in this experiment was E. coli HB101 (Maniatis et al., 1982) and the donor cell was E. coli No. 22 (The predominant isolated blaCTX-M producing Gram's negative bacteria in this study). The strains that exhibited resistance to any of the tested antibiotics were examined for the ability to transfer resistance by conjugation. The recipient and donor strains in vitro were grown to mid-log phase. Equal volumes of donor and recipient cells were spread on a Mueller-Hinton plate and incubated for $24 \mathrm{~h}$ at $37^{\circ} \mathrm{C}$. Positive and negative controls were prepared and examined. The resulting biomass was harvested, plated on Luria-Bertani agar 
Table 1. The sequences and sizes of the used primers.

\begin{tabular}{|c|c|c|c|}
\hline Primer & Forward & Reverse & PCR condition \\
\hline $\begin{array}{l}\text { blaTEM (858 bp) } \\
\text { (Arlet et al., 1995) } \\
\\
\text { bla CTX-M (499 bp) } \\
\text { (Johann et al., 2004) }\end{array}$ & $\begin{array}{l}\text { 5'- ATG AGT ATT CAA } \\
\text { CAT TTC CG -3' }\end{array}$ & $\begin{array}{l}\text { 5'- CCA ATG CTT ATT } \\
\text { CAG TGA GG-3' }\end{array}$ & $\begin{array}{l}95^{\circ} \mathrm{C} \text { for } 5 \mathrm{~min}, \text { followed by } 30 \text { cycles. } \\
\text { Denaturation: } 94^{\circ} \mathrm{C} \text { for } 30 \mathrm{~s} \text {. Annealing: } 55^{\circ} \mathrm{C} \\
\text { for } 1 \mathrm{~min} \text {. Extension: } 70^{\circ} \mathrm{C} \text { for } 1 \mathrm{~min} \text {. Final } \\
\text { extension: } 75^{\circ} \mathrm{C} \text { for } 10 \mathrm{~min} \text {. }\end{array}$ \\
\hline $\begin{array}{l}\text { bla SHV (827 bp) } \\
\text { (Al Naiemi et al., 2005) }\end{array}$ & $\begin{array}{l}\text { 5'- CTG GGA AAC } \\
\text { GGA ACT GAA TG-3' }\end{array}$ & $\begin{array}{l}5^{\prime} \text {-GGG GTA TCC } \\
\text { CGC AGA TAA AT-3' }\end{array}$ & $\begin{array}{l}95^{\circ} \mathrm{C} \text { for } 5 \mathrm{~min} \text {, followed by } 32 \text { cycles. } \\
\text { Denaturation: } 94^{\circ} \mathrm{C} \text { for } 1 \mathrm{~min} \text {. Annealing: } 57^{\circ} \mathrm{C} \\
\text { for } 1 \mathrm{~min} \text {. Extension: } 70^{\circ} \mathrm{C} \text { for } 1 \mathrm{~min} \text {. Final } \\
\text { extension: } 72^{\circ} \mathrm{C} \text { for } 10 \mathrm{~min} \text {. }\end{array}$ \\
\hline
\end{tabular}

plates (LB) containing streptomycin $(16 \mathrm{mg} / \mathrm{ml})$ and ceftazidime (8 $\mathrm{mg} / \mathrm{ml}$ ), and incubated for $24 \mathrm{~h}$ at $37^{\circ} \mathrm{C}$ (Sigma, USA). E. coli HB101 showed resistance to Streptomycin (recipient cell) while $E$. coli No. 22 was sensitive to Streptomycin (donor cell). However, E. coli No. 22 was resistant to ceftazidime while E. coli HB101was sensitive to ceftazidime.

\section{RESULTS}

\section{Bacterial isolates}

During the period from November 2009 to April 2010, 99 out of 560 clinically significant bacterial strains belonging to the family Enterobacteriaceae were resistant to most of the 15 different antibiotic discs including the third generation cephalosporins. The multi-resistant isolates were collected from urine catheter, wound, sputum, blood and different samples. The ESBL infection rate in the hospitalized patients (62\%) was higher than out-patients and most of them were in the intensive care unit (data are not shown).

\section{Identification of the bacterial isolates}

The red (lactose) and colorless (non-lactose) fermenting colonies on MacConkey agar or creamy (mucoid) colonies on blood agar were selected for gram stain, oxidase test and analyzed in VITEK 2 for bacterial identification. Gram's stained films showed gram's negative bacilli and oxidase test was negative for the lactose fermenting colonies and positive for non-lactose fermenting colonies. The number of the multi-resistant isolates was identified as $E$. coli (54 out of 358 ), $K$. pneumoniae (18 out of 96), $P$. aeruginosa (11 out 58), $P$. mirabilis (6 out of 17), E. cloacae (5 out of 18), $A$. baumanii (4 out of 9 ) and $E$. aerogenes (one out of 4 ). Most of the multi-resistant gram negative bacteria were isolated from urine samples (465 out of 560), whereas, blood samples were the lowest one (11 out of 560).

\section{Antimicrobial susceptibility test}

The isolated gram negative bacteria (Table 2) were resistant to the first, second and third generation of cephalosporins; CZL, CXM, CAZ, CTX and CRO. The antibiotic sensitivity of tigecycline (TGC), amikacin (AK), nitrofurantion (NI), gentamicin (GM), tazocin (PTZ), Amoxicillin / clavulanic acid (AUG) and ciprofloxacin (CIP) reached 84.8, 77.7, 42.4, 43.4, 71.7, 22.2 and $21.2 \%$, respectively. All the multiresistant gram negative bacteria were sensitive to MEM (86.8\%) except the isolates of $P$. aeruginosa and two isolates of $A$. baumanii. The antibiotic susceptibility of $E$. coli to TGC, AK, PTZ, $\mathrm{NI}, \mathrm{GM}, \mathrm{AUG}$ and CIP reached 98, 96, 89, 72, 63, 39 and $18.5 \%$, respectively. The antibiotic susceptibility rate of $K$. pneumoniae showed $100 \%$ against TGC, $83 \%$ against PTZ, $66.6 \%$ against AK, 28\% against CIP, 22\% against GM, $16.5 \%$ against $\mathrm{NI}$ and $5.5 \%$ against AUG. MEM and AUG were the effective antibiotics against $E$. coli and $K$. pneumoniae, but not active against other gram negative 
Table 2. The antibiotic activity (\%) on the multi-resistant isolates.

\begin{tabular}{|c|c|c|c|c|c|c|c|c|c|}
\hline \multirow{2}{*}{ Bacteria } & \multicolumn{9}{|c|}{ Antibiotic } \\
\hline & MEM & TGC & AK & NI & GM & PTZ & AUG & CIP & TS \\
\hline E. coli $(\mathrm{n}=54)$ & 100 & 98 & 96 & 72 & 63 & 89 & 39 & 18.5 & 20.3 \\
\hline K. pneumoniae $(\mathrm{n}=18)$ & 100 & 100 & 66.6 & 16.5 & 22 & 83 & 5.5 & 28 & 11.1 \\
\hline$P$. aeruginosa $(\mathrm{n}=11)$ & 0 & 0 & 9 & 0 & 9 & 9 & 0 & 9 & 0 \\
\hline Pr. mirabilis $\quad(n=6)$ & 100 & 100 & 100 & 0 & 0 & 66.6 & 0 & 0 & 0 \\
\hline A. baumanii $(\mathrm{n}=4)$ & 50 & 25 & 50 & 0 & 50 & 50 & 0 & 25 & 50 \\
\hline E. cloacae $\quad(\mathrm{n}=5)$ & 100 & 100 & 80 & 0 & 80 & 40 & 0 & 80 & 60 \\
\hline E. aerogenes $(n=1)$ & 100 & 100 & 0 & 0 & 0 & 0 & 0 & 0 & 0 \\
\hline Total $(\mathrm{n}=99)$ & 86.8 & 84.8 & 77.7 & 42.4 & 43.4 & 71.7 & 22.2 & 21.2 & 18.2 \\
\hline
\end{tabular}

AUG, Amoxicillin / clavulanic acid; CIP, ciprofloxacin; TS, Trimethoprime / sulfamethoxazole; MEM, meropenam; PTZ, tazocin; NI, nitrofurantion; AK, amikacin; GM, gentamicin; TGC, tigecycline; $n$, number of isolates.

Table 3. The phenotypic and molecular characterizations of the isolated ESBL producing gram negative bacteria.

\begin{tabular}{lccccccc}
\hline \multirow{2}{*}{ Organism } & \multirow{2}{*}{ VITEK } & \multirow{2}{*}{$\begin{array}{c}\text { D.D.S.T. } \\
\text { AMC/CAZ/CTX }\end{array}$} & \multicolumn{2}{c}{ E-test } & \multicolumn{3}{c}{ PCR } \\
\cline { 4 - 8 } & & 53 & 53 & 53 & TEM & CTX-M & SHV \\
\hline E. coli $(\mathrm{n}=54)$ & 53 & 18 & 18 & 18 & 15 & 42 & 37 \\
K. pneumoniae $(\mathrm{n}=18)$ & 18 & 2 & 1 & 5 & 1 & 9 & 12 \\
E. cloacae $(\mathrm{n}=5)$ & 0 & 1 & 1 & 1 & 1 & 0 & 0 \\
E. aerogenes $(\mathrm{n}=1)$ & 0 & 6 & 6 & 6 & 3 & 1 & 1 \\
P. mirabilis $(\mathrm{n}=6)$ & 0 & 80 & 79 & 83 & 40 & 53 & 51 \\
Total $(\mathrm{n}=84)$ & 71 & 95 & 94 & 98.8 & 47.6 & 63.1 & 60.7 \\
Total $(\%)$ & 84.5 & & & & & &
\end{tabular}

isolates. The isolates of $P$. aeruginosa were resistant to the tested antibiotics except CIP, PTZ, AK and GM, but MEM and TGC have antimicrobial activities against $E$. aerogenes.

\section{Phenotypic detection of ESBLs}

The VITEK2 (Table 3) detected the presence of ESBL activity of the tested isolates $(71 \%)$ represented by $K$. pneumoniae (100\%) and E. coli (98\%). Using DDST, the enhanced inhibition zone of the ceftazidime or cefotaxime in combination with clavulanic acid detected the presence of ESBL activity by 80 isolates (81\%). The rate of ESBL detection reached $100 \%$ by $K$. pneumoniae, $P$. mirabilis and $E$. aerogenes, whist $98 \%$ by $E$. coli and E. cloacae. The production of ESBL by DDST was not detected in one isolate of $E$. coli and three isolates of $E$. cloacae. On the other hand, the synergic activity of clavulanic acid combined with ceftazidime (TZ) or cefotaxime (CT) was confirmed by two different E-test strips containing ceftazidime and cefotaxime with or without clavulanic acid. The ceftazidime ESBL strips (TZ/TZL) detect the presence of ESBL activity by $80 \%$ of the tested isolates. Furthermore, the rate of the ESBL detection was $100 \%$ by $K$. pneumoniae, P. mirabilis and E. aerogenes; $98 \%$ by E. coli; and $20 \%$ by E. cloacae. The production of ESBL was not detected by one isolate of $E$. coli and four isolates of $E$. cloacae. With cefotaxime ESBL strips (CT/CTL), ESBL activity was detected by all the tested isolates $(84 \%)$ represented by $100 \%$ in K. pneumoniae, P. mirabilis, E. aerogenes, E. cloacae and $98 \%$ in E. coli. The production of ESBL by ceftazidime ESBL strips was not detected in one isolate of $E$. coli. The production of ESBL was not recorded by all the tested $P$. aeruginosae and $A$. baumanii isolates in this study.

The analysis of the phenotypic ESBLs showed that there are differences between the VITEK 2 and both of the E-test and DDST. Out of 99 tested multiresistant isolates, 71 isolates recorded ESBL production by VITEK 2 according to two species. On the other hand, 79 isolates were ESBL producers by TZITZL, 83 isolates were ESBL producers by CT\CTL and 80 isolates were ESBL producers by DDST according to five species. 


\section{Genotypic detection of ESBLs}

The PCR products that were amplified by specific primers of ESBL were detected in 75 isolates out of 84 tested ESBL producing gram negative bacteria with $89.3 \%$. The blaTEM genotypes (858 bp) were detected in 40 isolates with $47.6 \%$ (Table 3 ). They were recorded in 20 isolates of $E$. coli with $37 \%, 15$ isolates of $K$. pneumoniae with $83.3 \%, 3$ isolates of $P$. mirabilis with $50 \%$, one isolate of E. cloacae with $20 \%$ and one isolate of $E$. aerogenes with $100 \%$. The blaCTX-M primers manifested 499 bp amplicon in 53 isolates with $63.1 \%$ of the 84 tested isolates. The blaCTX-M genotypes were reported in 42 isolates of $E$. coli with $78 \%, 9$ isolates of $K$. pneumoniae with $50 \%$, one isolate of $P$. mirabilis with $17 \%$ and one isolate of $E$. aerogenes with $100 \%$. All the isolates of $E$. cloacae did not detect the blaCTX-M gene. The blaSHV primers detected the characteristic of $827 \mathrm{bp}$ amplicon in 51 isolates out of the 84 tested bacteria by $60.7 \%$. The blaSHV genotypes were detected in 37 isolates of $E$. coli with $68.5 \%, 12$ isolates of $K$. pneumoniae with $66.6 \%$, one isolate of $P$. mirabilis with $17 \%$ and one isolate of $E$. aerogenes with $100 \%$. They were not detected in all isolates of E. cloacae. Overall, the blaCTX-M was the commonest genotype $(63.1 \%)$ followed by blaSHV $(60.7 \%)$ and blaTEM (47.6\%). The most predominant ESBL producer was E. coli $(62 \%)$ followed by $K$. pneumoniae $(20.2 \%)$. The blaSHV, blaTEM and blaCTX$\mathrm{M}$ genotypes in combination were present in 18 isolates with $21.4 \%$. They were detected in K. pneumoniae $(6$ isolates), $E$. coli (11 isolates) and one isolate of $E$. aerogenes. On the other hand, eight isolates out of the 84 bacteria confirmed the presence of blaSHV and blaTEM combination in $K$. pneumoniae (4 isolates), $E$. coli (3 isolates) and one isolate of $P$. mirabilis. In addition, the blaSHV and blaCTX-M enzymes in combination were produced by 18 isolates of $E$. coli. Four isolates of $E$. coli and 3 isolates of K. pneumoniae produced ESBL by both blaTEM and blaCTX-M enzymes. The amplified PCR product for each blaTEM, SHV and blaCTX-M genes of the four ESBL producing gram negative bacteria were selected for sequencing according to the resulting antibiotic susceptibility test, phenotypic and genotypic characterization (Figures 1, 2, 3 and 4). Agarose gel electrophoresis results show that all the four selected ESBL producing isolates had identical banding patterns in accordance to the tested primers (Figures 5 and 6).

From Table 4, the forward and reverse DNA nucleotide sequences of the blaTEM producing $P$. mirabilis (No. 54) are identified as blaTEM producing $P$. mirabilis from gene bank No. JN043376 (78 to 99\%). The nucleotide sequences of DNA forward and reverse revealed that the blaCTX-M producing $E$. coli (No. 22) is identified as blaCTX-M of E. coli gene bank No. FJ668785 (99\%). The forward and the reverse DNA nucleotide sequences of the blaSHV producing $K$. pneumoniae (No. 93) are identical to blaSHV producing $K$. pneumoniae gene bank No. FJ815288 and HM002660, respectively (98\%). The forward and reverse DNA nucleotide sequences of the bla-TEM producing E. cloacae (No. 21) are identified as blaTEM producing Enterobacter sp. gene bank No. FJ349257 (91\%) and bla-TEM producing E. cloacae gene bank No. AY302260 (90\%), respectively. The identical band patterns of the selected ESBL isolates were identified by $16 \mathrm{SrDNA}$ at a molecular size of $1500 \mathrm{bp}$. The resulting DNA sequences of the selected ESBL isolates including F27 and R1492 primers were deposited by the gene bank nucleotide sequence database (Table 5).

The gene bank accession numbers of the forward and reverse ESBL producing $P$. mirabilis (No. 54) are Banklt1534618 JX17256 and Banklt1534627 JX17257, respectively. Banklt1534363 JX017250 and Banklt1534367 JX017251 were the gene bank accession numbers for the forward and reverse ESBL producing $E$. coli (No. 22), respectively. Banklt1534372 JX17252 and Banklt1534380 JX17253 were the gene bank accession numbers for the forward and reverse ESBL producing $K$. pneumoniae (No. 93), respectively. Banklt1534608 JX17254 and Banklt1534614 JX17255 were the gene bank accession numbers of the forward and reverse ESBL producing E. cloacae (No. 21), respectively.

\section{Conjugation assay}

Based on the conjugation assay in this experiment between the recipient cell (E. coli HB101) and the donor cell ( $E$. coli No. 22), the blaCTX-M gene was able to be transferrable to transconjugant successfully suggesting that they were plasmid mediated. The gene bank numbers of the resulting DNA sequences of the forward and reverse CTX primers for the transconjugant were identified as bla-CTX-M producing $E$. coli genes bank No. FJ668785 (99\%) similar to the forward and reverse DNA nucleotide sequences of the bla-CTX-M producing E. coli (No. 22).

\section{DISCUSSION}

Eighty four (84) out of 560 isolates are ESBL producing gram negative bacteria including E. coli, K. pneumniae, $P$. mirabilis, E. cloacae and $E$. aerogenes. The specimens were collected from different departments while urine samples were from elderly females patients above 50 years old having urinary tract infections. The ESBL infection rate in the hospitalized patients (62\%) is higher than the out-patients mainly in the intensive care unit. These results are correlated with that obtained by 


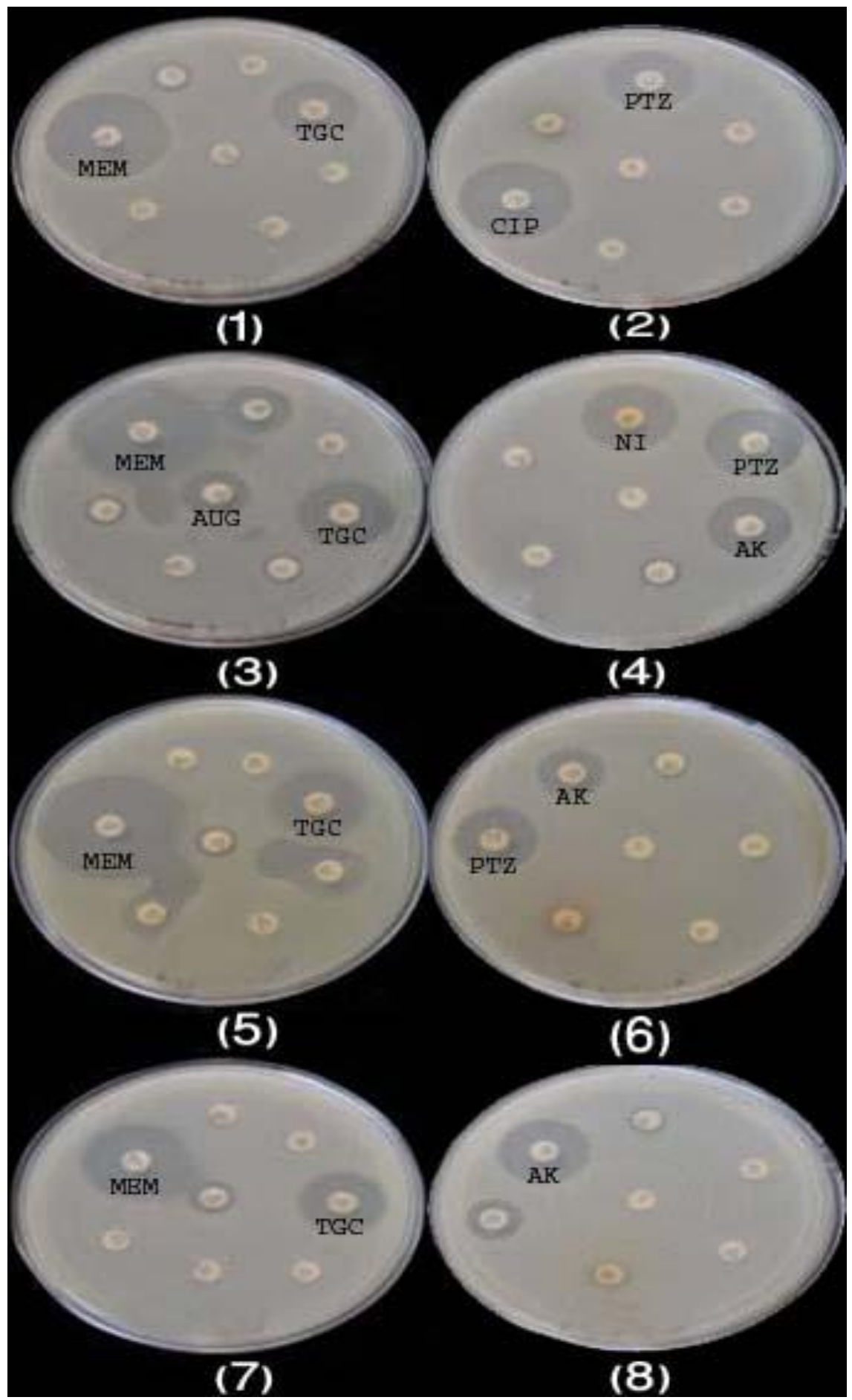

Figure 1. The antibiotic activity of the selected multi-resistant isolates. Plates 1 and 2 , E. cloacae (No. 21); 3 and 4, E. coli (No. 22); 5 and 6, P. mirablis (No. 54); 7 and 8, K. pneumoniae (No. 93).

Kiratisin et al. (2008) where a total of 2,777 patients were identified as having infections due to $E$. coli or $K$. pneumoniae at both major tertiary-care centers. The majority of patients were over 60 years old and most of 


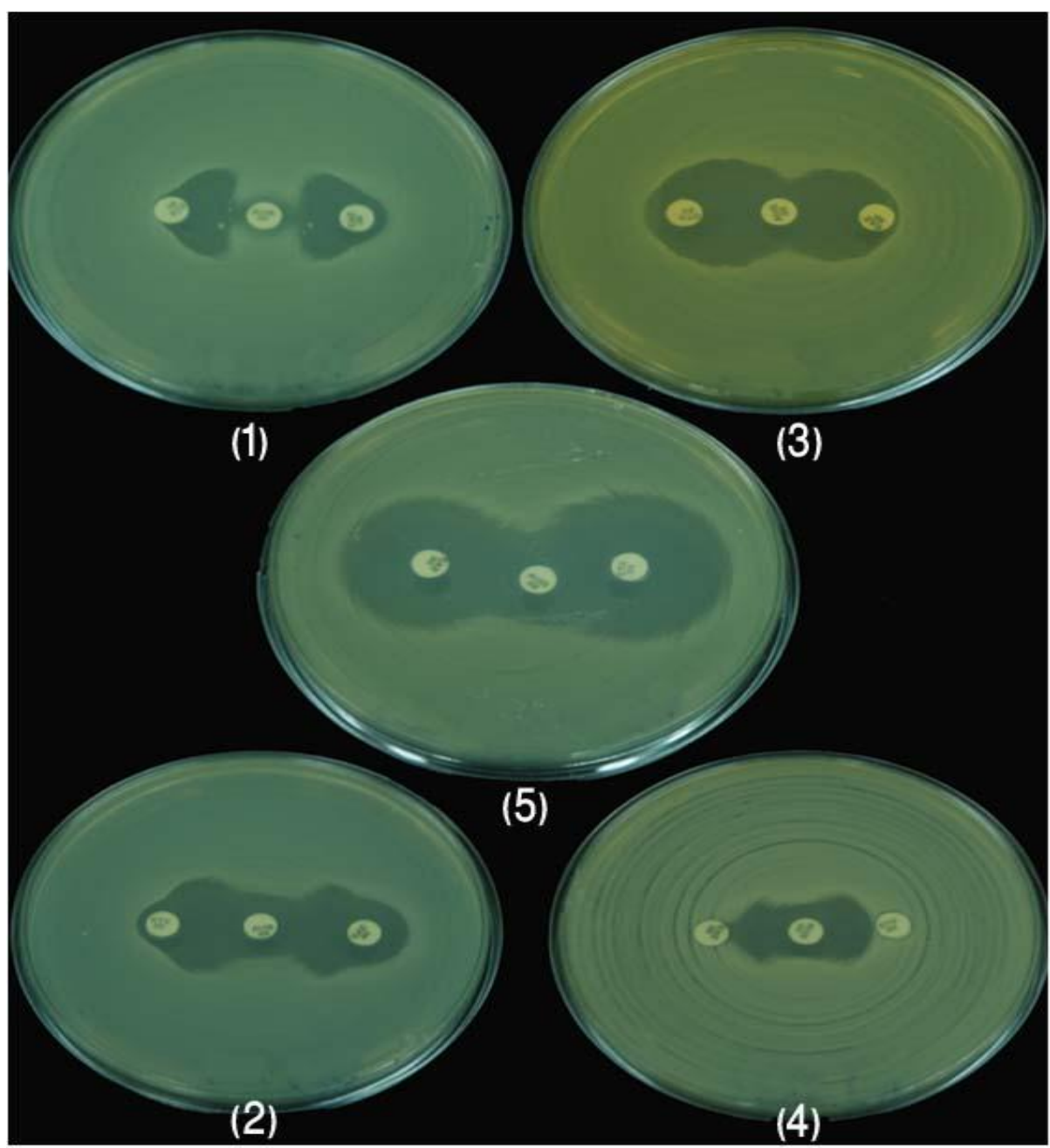

Figure 2. The enhancement bacterial zone of the selected ESBL isolates by DDST. Plate 1, E. cloacae (No. 21); 2, E. coli (No. 22); 3, P. mirablis (No. 54); 4, K. pneumoniae (No. 93); 5, E. coli ATCC No. 25922. Left disc: CAZ; middle disc: AUG; right disc: CTX.

them had been hospitalized in the intensive care units. All the ESBL producers were recovered from urine specimens and female patients were the predominant for particularly ESBL producing $E$. coli infection. Our results are in agreement with those of Dechen et al. (2009) who identified $81 \mathrm{ESBL}$ producing isolates out of 238 Gram's negative bacilli by DDST and phenotypic confirmatory test. The isolates were E. coli $(\mathrm{n}=34), K$. pneumoniae ( $\mathrm{n}$ $=20), P$. aeruginosa $(\mathrm{n}=15), P$. mirabilis $(\mathrm{n}=3)$, Morganella morganii $(\mathrm{n}=5)$ and Citrobacter frundii $(\mathrm{n}=$ 4). The isolates were collected from 152 urine, 70 wound, 12 blood, 22 sputum and 2 cerebrospinal fluid samples. It was concluded that ESBL producers can be detected by DDST and phenotypic confirmatory test with equal efficacy. Data has shown that VITEK 2 GNI-GNB system reported all the members of the tested gram negative bacilli successfully. E. coli were the predominant gram's negative bacilli among the others and MEM was the only susceptible antibiotic against all the multi-resistant gram negative isolates.

Caroline and Michael (2003) demonstrated that the VITEK 2 instrument has an accuracy of $93.0 \%$ for the identification of gram's negative bacilli. Khalid et al. (2009) reported that out of a total of 11,886 isolated gram negative bacilli, 2695 were ESBL producers. E. coli and $K$. pneumoniae were the predominant comparatively in the hospital wards while Proteus spp. was predominant in medical wards. Urine was the major source with low occurrence in blood cultures. Shah and Mulla (2012) found that MEM and ETP were effective against ESBL producers especially $E$. coli and $K$. pneumoniae and remain good choices for the treatment of suspected 


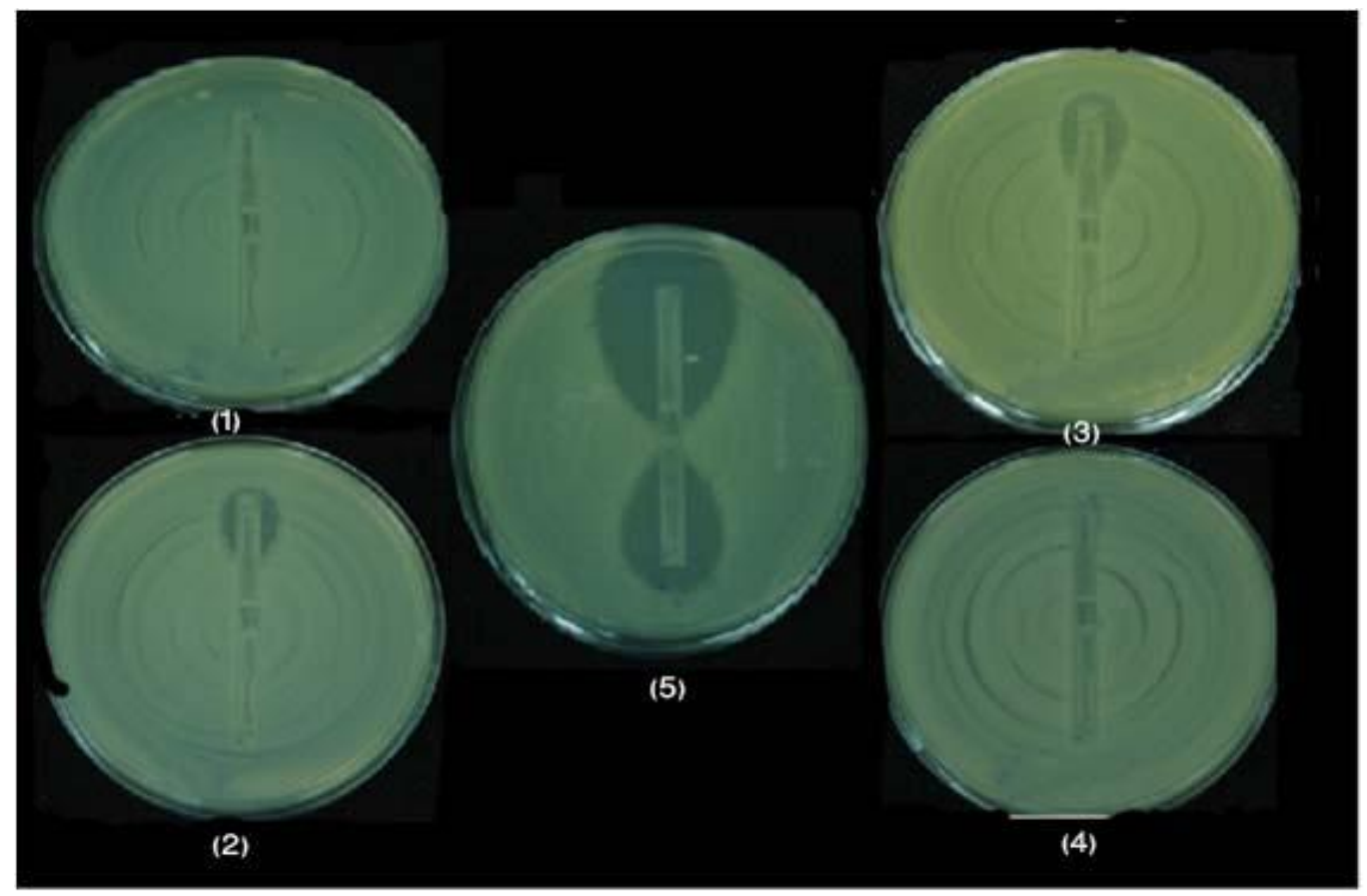

Figure 3. The phenotypic determination of the selected ESBL isolates by E-test strips (CT/CTL Plate 1, E. cloacae (No. 21); 2, E. coli (No. 22); 3, P. mirablis (No. 54); 4, K. pneumoniae (No. 93); 5, E. coli ATCC No. 25922.

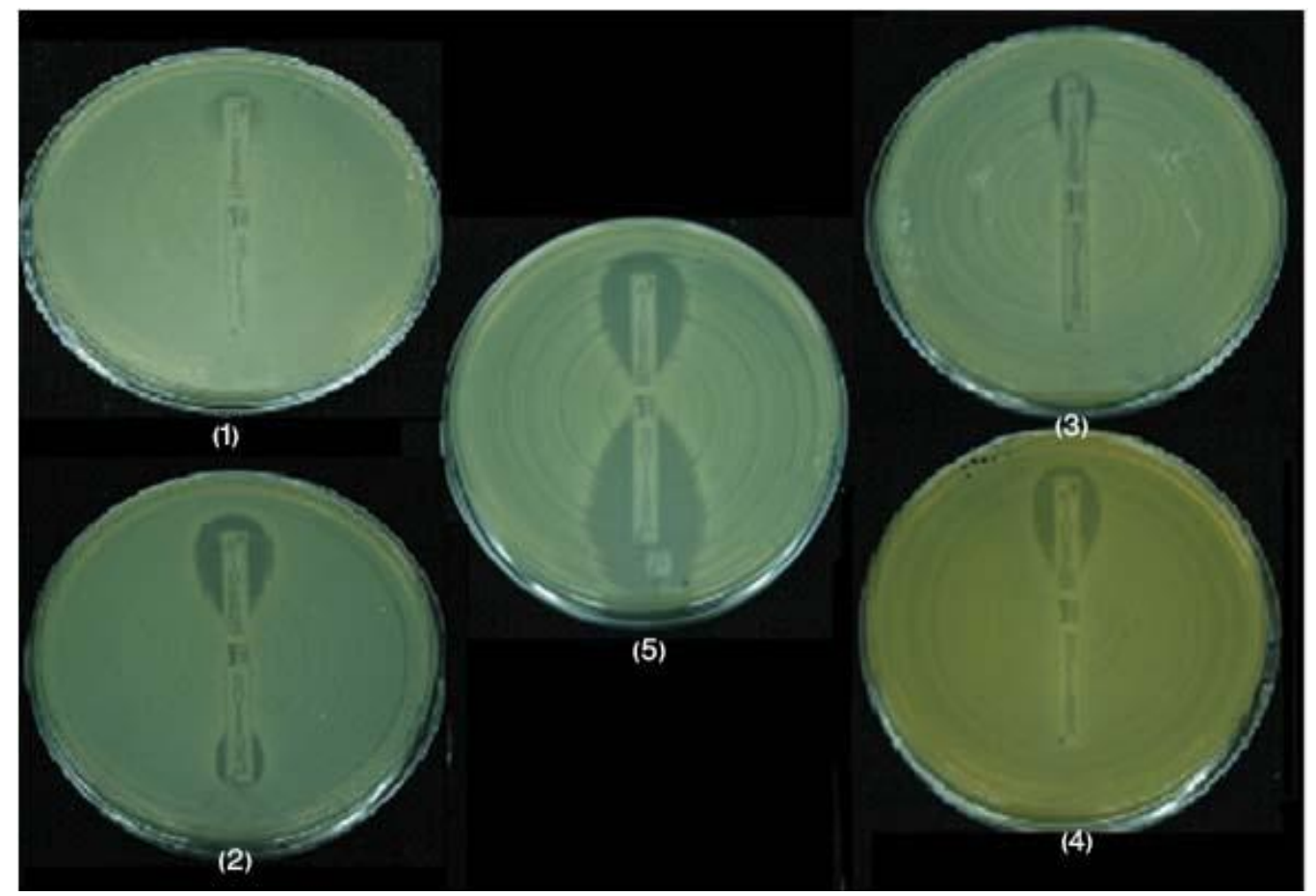

Figure 4. The phenotypic determination of the selected ESBL isolates by E-test strips (TZ/TZL). Plate 1, E. cloacae (No. 21); 2, E. coli (No. 22); 3, P. mirablis (No. 54); 4, K. pneumoniae (No. 93); 5, E. coli ATCC No. 25922. 


\section{blaSHV(827bp)}

\section{blaTEM(858bp)}

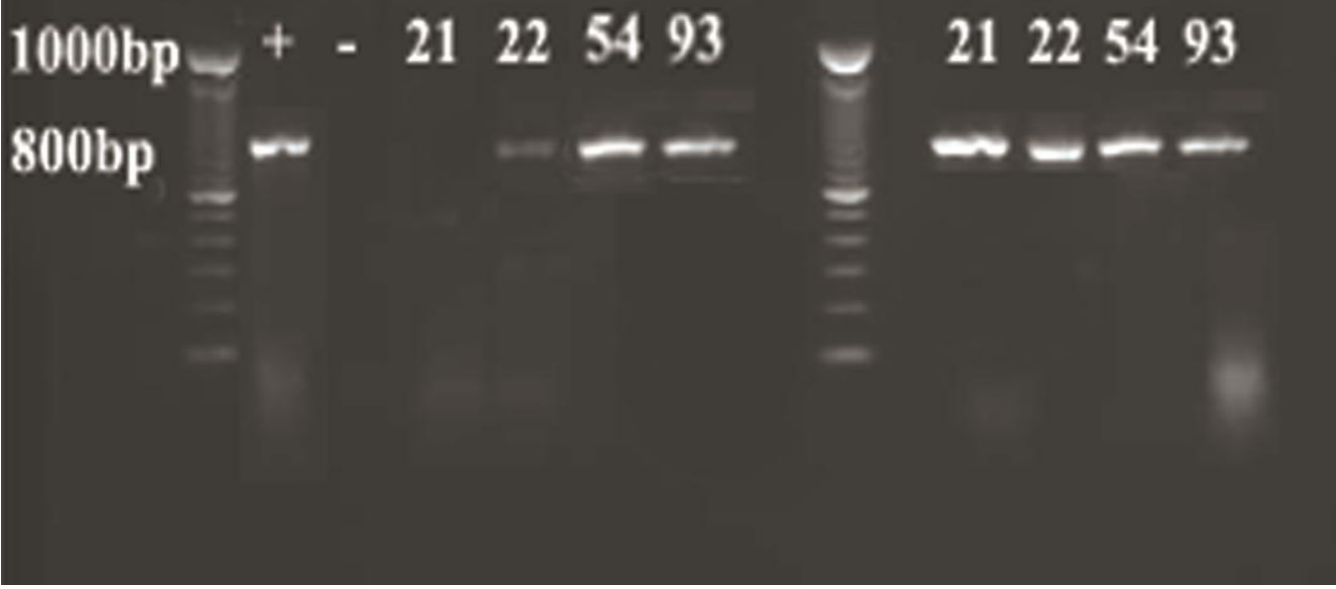

Figure 5. The agarose gel electrophoresis showing the identical band patterns of the blaTEM (858 bp) and blaSHV (827 bp) produced by E. cloacae (No. 21), E. coli (No. 22), P. mirablis (No. 54) and $K$. pneumonia (No. 93). Negative band of blaSHV is seen by E. cloacae (No. 21).

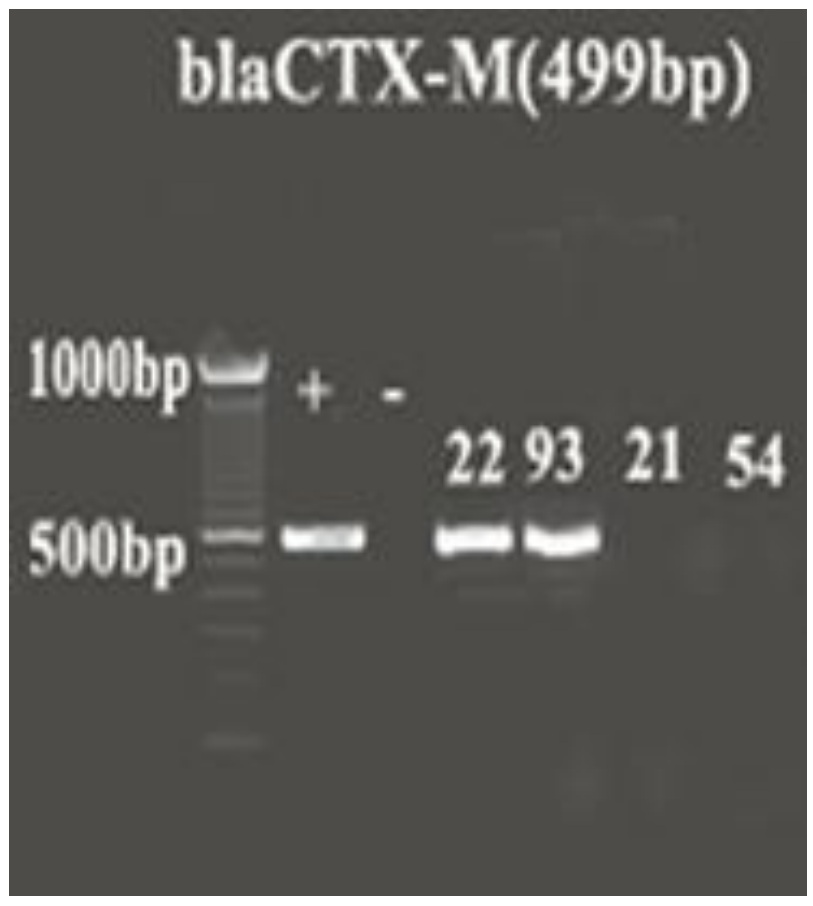

Figure 6. The agarose gel electrophoresis showing the identical band patterns of the blaCTX-M (499 bp) produced by E. coli (No. 22) and K. pneumoniae (No. 93). Negative bands of blaCTX-M are detected by E. cloacae (No. 21) and P. mirablis (No. 54). 
Table 4. The analysis of the DNA sequences of the ESBL primers using NCBI/BLAST.

\begin{tabular}{|c|c|c|c|}
\hline Organism & ESBL primer & Forward ESBL gene bank no. & Reverse ESBL gene bank number \\
\hline \multirow{2}{*}{ E. coli ( No. 22) } & \multirow{2}{*}{ CTX-M } & FJ668785 & \\
\hline & & \multicolumn{2}{|c|}{ Escherichia coli strain 61 plasmid bla-CTX-M15 gene. Identity $99 \%$. } \\
\hline & & FJ815288 & HМ002660 \\
\hline K. pneumoniae ( No. 93) & SHV & $\begin{array}{l}\text { Klebsiella pneumonia strain HB100 plasmid } \\
\text { bla-CTX-M15 gene. Identity } 98 \% \text {. }\end{array}$ & $\begin{array}{l}\text { Klebsiella pneumonia } 379 T \text { ESBL CTX- } \\
\text { M15 gene. Identity } 98 \% \text {. }\end{array}$ \\
\hline \multirow{2}{*}{ P. mirabilis (No. 54) } & \multirow{2}{*}{ TEM } & \multicolumn{2}{|c|}{ JN043376 } \\
\hline & & \multicolumn{2}{|c|}{ Proteus mirabilis Strain M-0928-11 ESBL TEM-1 gene Identify by $78-99 \%$. } \\
\hline & & FJ349257 & AY30226 \\
\hline E. cloacae (No. 21) & TEM & $\begin{array}{l}\text { Enterobacter species ESBL TEM-150 } \\
\text { gene. Identity } 91 \% \text {. }\end{array}$ & $\begin{array}{l}\text { Enterobacter cloacae strain } 212 \text { TEM-1 } \\
\text { gene. Identity } 90 \% \text {. }\end{array}$ \\
\hline
\end{tabular}

ESBL infections. In the present study, the detection of ESBLs varies between VITEK 2 (84.5\%), TZ/TZL (94\%), CT/CTL (98.8\%) and DDST (95\%) on 560 isolates of $E$. coli, K. pneumoniae, $P$. aeruginosa, $P$. mirabilis, $E$. cloacae, $E$. aerogenes and $A$. baumanii. The performance characteristics of the conventional methods for the detection of ESBLs (E-test and DDST) were better than the automated system (VITEK2). This finding is in agreement with those of Irith et al. (2007) who compared commercially microbiological identification testing system (VITEK 2) with the conventional phenotypic confirmatory tests (E-test and DDST) to detect ESBL production in gram's negative bacteria. The E-test showed the highest specificity for the detection of ESBLs followed by DDST and VITEK2. They recommended the use of a manual test for confirmation once an organism is reported positive for ESBL production by any of the semiautomated system. Our results also agree with those of Maria et al. (2011) who evaluated the accuracy of positive ESBL results by VITEK 2 regarding clinical isolates of $E$. coli and K. pneumoniae using DDST and Etest displaying distinct results by which the VITEK 2 system was in disagreement in $23.9 \%$ of cases with DDST and in $15.3 \%$ with E-test.

Molecular detection in the present work showed that the tested isolates are positive ESBL produced by blaSHV, blaCTX-M and blaTEM genotypes. The blaCTX-M is the most predominant ESBL gene $(63.1 \%)$ followed by blaSHV (60.7\%) detected in E. coli $(62 \%)$ and K. pneumoniae (20.2\%). Two or more genotypes of ESBL were present in 34 isolates of E. coli, blaCTX-M and blaSHV being the most common combination genes (33.3\%) followed by bla-SHV with bla-TEM and bla-CTX$M(11 \%)$. ESBL were present in 17 isolates of $K$. pneumoniae, bla-SHV and bla-TEM being the most combination genes (39\%) followed by blaSHV with blaTEM and blaCTX-M (28\%). This finding is in agreement with those of Ankur et al. (2009) who examined ESBL production phenotypically for a total of 200 consecutive clinical isolates of $E$. coli $(n=143)$ and $K$. pneumoniae $(n=57)$ collected at a tertiary care hospital followed by further typed for the blaTEM / SHV / CTX-M genes by PCR using specific primers. ESBLs were found in $63.6 \%$ of $E$. coli isolates and $66.7 \%$ of $K$. pneumoniae isolates and majority of them harboured two or more ESBL genes (57.3\%). Overall, blaCTX-M was the commonest genotype $(85.4 \%)$ followed by blaTEM $(54.9 \%)$ and blaSHV $(32.9 \%)$ either alone or in combination. Two or more genes for ESBL were present in 47 out of 82 ESBL isolates including the blaTEM with blaCTX-M being the most common combination (28.1\%). They concluded high ESBL occurrence with CTX-M as the emerging type in the selected hospital. The results in the present study identified successfully the selected ESBL producing gram negative bacteria by 16 SrDNA. Identification of bacteria by $16 S$ rDNA gene sequences analysis in the present work discriminate more finely among the selected strains than is possible with phenotypic methods. Thus, it provides an accurate identification at the species level and can clarify the clinical importance of the isolated bacteria of the infectious diseases (Fredricks and Relman, 1996; Clarridge et al., 2001).

Tang et al. (2000) compared a variety of identification systems including cellular fatty acid profiles, carbon source utilization and conventional biochemical identification with the 16SrRNA gene sequence to evaluate both unusual aerobic gram negative bacilli isolated from clinical specimens. They found that 16SrRNA gene sequence provided more rapid, unambi- 
Table 5. The characteristics and identifications of the selected ESBL gram negative bacteria by $16 \mathrm{~S}$ rDNA.

\begin{tabular}{|c|c|c|c|c|c|c|c|c|}
\hline \multirow{2}{*}{ Organism } & \multicolumn{3}{|c|}{ Phenotypic test } & \multicolumn{3}{|c|}{ PCR } & \multicolumn{2}{|c|}{ Gene bank accession number (16S rDNA) } \\
\hline & VITEK & E-Test & DDST & TEM & CTX-M & SHV & F27 & R1492 \\
\hline E. coli (No. 22) & + & + & + & + & + & + & Banklt1534363 JX017250 & Banklt1534367 JX017251 \\
\hline K. pneumoniae (No. 93) & + & + & + & + & + & + & Banklt1534372 JX017252 & Banklt1534380 JX017253 \\
\hline P. mirabilis (No. 54) & - & + & + & + & - & + & Banklt1534618 JX017256 & Banklt1534627 JX017257 \\
\hline E. cloacae (No.21) & - & + & + & + & - & - & Banklt1534608 JX017254 & Banklt1534614 JX017255 \\
\hline
\end{tabular}

guous identification of the difficult bacterial isolates than did conventional methods and that this identification could translate to improve clinical outcomes.

Bosshard et al. (2003) found that only a minority of the clinical laboratory isolates of aerobic gram negative rods could be correctly identified by phenotypic methods whereas 16SrRNA gene sequencing is an excellent method for identifying these organisms which are difficult to identify by conventional methods. Our results show that the blaCTX-M genotype of $E$. coli (No. 22) is able to be transferred to transconjugant successfully when conjugated with E. coli (HB101); this suggests that they are plasmid mediated.

Iroha et al. (2010) reported that ESBLs are carried on bacterial chromosomes or plasmids and plasmid-mediated ESBLs can carry genes on them that have the ability to transfer a replica of themselves to other bacteria. They also can carry genes conferring resistance to other classes of antibiotics that make the recipient bacteria resistant to multiple antibiotics. Furthermore, these plasmids can emerge on strains that do not cause human diseases and then the nonpathogenic strains could transfer their plasmids to strains that can cause human diseases.

Plasmid conjugation is an important mechanism of disseminating drug resistance among bacteria population. In conclusion, the prevalence rate of ESBL producing organisms is high globally. The ESBL producing organisms are known to cause serious nosocomial infections, long term carriage in the community, community-acquired infections such as urinary tract infections and intraabdominal abscess. The findings from the present study reveal high prevalence of ESBL from Ahmadi hospital in Kuwait where E. coli is the highest producer followed by $K$. pneumoniae, $P$. mirabilis, E. cloacae and $E$. aerogenes, respectively. ESBL producers can be detected by E-test and DDST with equal efficacy. The blaCTX $M$ is the most predominant ESBL genotypes among the multiresistant gram negative bacteria including the third generation of cephalosporins. Thus, this study emphasizes the inclusion of ESBL detection in routine laboratory tests in hospitals and clinics especially in the developing countries.

\section{ACKNOWLEDGEMENT}

The authors wish to thank Professor Dr. Ali A. Dashti, Professor of Microbiology, Medical Laboratory Science Department, Faculty of Allied
Health Science, Health Science Center, Kuwait University, Kuwait, for his excellent technical assistance.

\section{REFERENCES}

Al Naiemi N, Duim B, Savelkoul P, Spanjaard L, Jongr E, Bart, A, Vandenbroucke C, Grauls D, Menno D (2005). Widespread Transfer of Resistance genes between Bacterial species in an Intensive Care Unit: Implication for Hospital Epidemiology. J. Clin. Microbiol. 34(9):4862-4864.

Ankur G, Prasad KN, Amit P, Sapna G, Ujjala G, Archana A (2009). Extended spectrum $\beta$-lactamase in Escherichia coli and Klebsiella pneumoniae, and associated risk factors. Indian J. Med. Res. 129:695-700.

Arlet G, Brami G, Decre D, Flippo A, Gaillot O, Lagrange PH Philippon A (1995). Molecular characterization by PCRrestriction fragment length polymorphism of TEM betalactamases. FEMS Microbiol. Lett. 134: 203-208.

Arlet G, Philippon A (1991). Construction by polymerase chain reaction and itragenic DNA probs for three main types of transferable $\beta$-lactamases (TEM, SHV, GARB). FEMS. Microbiol. lett. 82:19-26.

Bauer AW, Kirby WM, Sherris JC, Turck M (1966). Antibiotic susceptibility testing by a standardized single disk method. Am. J. Clin. Pathol. 45:493-496.

Bauernfeind A, Casellas JM, Goldberg M, Holley M, Junwirth R, Mongold P, Rohnush T, Schweighart S, Wilhelm R (1992). A new plasmidic cefotaximase from patients infected with Salmonella typhimurium. Infect. 20:158-163.

Bonnet R (2004). Growing group of extended-spectrum $\beta$ lactamases: the CTX-M enzymes. Antimicrob. Agents Chemother. 48(1):1-14 
Bosshard PP, Abels S, Zbinden R, Bottgar EC, Altwegg M (2003). Ribosomal DNA sequencing for identification of aerobic Grampositive rods in the clinical laboratory (an 18-month evaluation). J. Clin. Microbiol. 4:4134-4140.

Bradford PA (2001). Extended spectrum $\beta$-lactamases in the 21st century: characterization, epidemiology and the detection of this important resistance threat. Clin. Microbiol.Rev. 14:933-951.

Bratu S, Landman D, Haag R, Recco R, Eramo A, Alam M, Quale J (2005). Rapid spread of carbapenem-resistant Klebsiella pneumoniae in New York City: a new threat to our antibiotic armamentarium. Arch. Intern. Med. 165(12):1430-1443.

Canton R, Perez-Zazquez M, Oliver A, Coque TM, Loza E, Ponz F, Baquero $F$ (2001). Validation of VITEK-2 and the advanced Expert System with a collection of Enterobacteriaceae harbouring extended spectrum or inhibition resistant $\beta$-lactamases. Diagn. Micr. Infec. Dis. 41:65-70.

Caroline M, Michael J (2003). Evaluation of the Vitek 2 ID-GNB Assay for identification of members of the family Enterobactericaea and other nonenteric Gram negative bacilli and comparison with the Vitek GNI cards. J. Clin. Microbiol. 41(5):2096-2101.

Clarridge JE, Attorri S, Musher DM, Hebert J, Dunbar S (2001). Streptococcus constellatus and Streptococcus anginosus ("Streptococcus milleri group") are of different clinical importance and are not equally associated with abscess. Clin. Infect. Dis. 32:15111515.

Clinical and Laboratory Standards Institute (CLSI) (2005). Performance standards for antimicrobial susceptibility testing: 15th informational supplememnt. CLSI document M100-S15. CLSI. Wayne, PA.

Cormican MG, Marchall SA, Jones RN (1996). Detection of extended spectrum $\beta$-lactamases (ESBL) producing strains by the E-test ESBL screen. J. Clin. Microbiol. 34(8): 1880-1884.

Dashti A, Jadan M, Abdulsamad A, Dashti H (2009). Heat treatment of bacteria: DNA extraction for molecular techniques. KMJ. 41(2):117122.

Dashti A, Jadaon M, Gomaa H, Noronha B, Udo E (2010). Transmission of Klebsiella pneumoniae clone harbouring genes for CTX-15-LIKE and SGAV-112 enzymes in a neonatal intensive care unit of a Kuwaiti hospital. J. Med. Microbiol. 59:687-692.

Dechen CT, Shyamasree D, Luna A, Ranabir P, Takhellambam SK (2009). Extended spectrum $\beta$-lactamase detection in Gram-negative bacilli of nosocomial origin. J. Global Infect. Dis. 1(2): 87-92.

Dhillon R, Clark J (2011). ESBL: A clear and present danger? Crit. Care Res. Pract. 2012 (2012):1-11.

Duttaroy B, Mehta S (2005). Extended spectrum $\beta$-lactamases (ESBL) in clinical isolates of Klebsiella pneumoniae and Escherichia coli. Indian J. Pathol. Micr. 48(1):45-48.

Fredricks DN, Relman DA (1996). Sequence-based identification of microbial pathogens: a reconsideration of Koch's postulates. Clin. MicrobioL. Rev. 9:18-33.

Irith W, Heinrich KG, Dietrich M, Enno S, Harald S (2007). Detection of extended-spectrum $\beta$-lactamase among Enterobacteriaceae by use of semiautomated microbiology system and manual detection procedures. J. Clin. Microbiol. 45(4):1167-1174.

Iroha IR, Amadi ES, Oji AE, Nwuzo AN, Ejike-Ugwa PC (2010). Detection of plasmid borne extended spectrum $\beta$-lactamase enzymes from blood and urine isolates of Gram negative bacteria from a University teaching hospital in Nigeria. Curr. Res. Bacteriol. 3(2):77-83.

Ivanova D, Markovska R, Hadjiera N, Mitov I, Bauernfeind A (2008). Extended spectrum $\beta$-lactamase-producing Serratia marcescens outbreak in a Bulgarian hospital. J. Hosp. Infect. 70:60-65.

Johann D, Pitout D, Ashfaque H, Nancy D (2004). Phenotypic and Molecular Detection of CTX-M- $\beta$-Lactamases produced by Escherichia coli and Klebsiella spp. J. Clin. Microbiol. 42(12):57155721.

Khalid MB, Abiola CS, Afaf EJ (2009). Prevelence of extendedspectrum $\beta$-lactamase-producing Enterobacteriacaeae in Bahrain. J. Infect. Public Health. 2:129-135.
Kiratisin P, Apisarnthanark A, Laesripa C, Saifon P (2008). Molecular Characterization and Epidemiology of extended-spectrum $\beta$. lactamase-producing Escherichia coli and Klebsiella pneumoniae isolates causing health care-associated infection in Thailand, where the CTX-M family is endemic. Antimicrob. Agents Ch. 52(8):28182824.

Knothe H, Shah P, Krcmery V, Antal M, Mitsuhashi S (1983).Transferable resistance to cefotaxime, cefoxitin, cefamandole and cefuroxime in clinical isolates of Klebsiella pneumoniae and Serratia marcescens. Infect.11:315- 317.

Kollef MH, Sherman G, Ward S, Fraser VJ (1999). Inadequate antimicrobial treatment of infections: a risk factor for hospital mortality among critically ill patients. Chest. 115:462-474.

Leflon-Guibout V, Jurand C, Bonacorsi S, Espinasse F, Guelfi MC, Duportail F, Heym B, Bingen E, Nicolas-Chanoine MH (2004). Emergence and spread of three clonally related virulent isolates of CTX-M-15-producing Escherichia coli with variable resistance to aminoglycosides and tetracycline in a French geriatric hospital. Antimicrob. Agents Chemother. 48(10):3736-3742.

Livermore DM (1995). $\beta$-lactamases in laboratory and clinical resistance. Clin. Microbiol. Rev. 8:557-584.

Lou YK, Qin H, Molodysky E, Morris BJ (1993). Simple microwave and thermal cycler boiling methods for preparation of cervicovaginal lavage cell samples prior to PCR for human papillomavirus detecteion. J. Virol Methods. 44:77-81.

Maniatis T, Fritsch EF, Sambrook J (1982). Molecular cloning: a laboratory manual. Cold Spring Harbor Laboratory, Cold Spring Harbor, N.Y.

Maria JE, Rita R, Manuela R, Acacio GR, Cidalia P (2011). Extendedspectrum $\beta$-lactamases of Escherichia coli and Klebsiella pneumoniae screened by the VITEK 2 system. J. Med. Microbiol. 60(6):756-760

McFarland J (1907). Nephelometer: an instrument for estimating the number of bacteria in suspensions used for calculating the opsonic index and for vaccines. J. Am. Med. Ass. 14:1176-1178.

Merk S, Meyer H, Greiser-Wilke I, Spraque LD, Neubauer H (2006). Detection of Burkholderia cepacia DNA from artificially EDTA-blood and lung tissue comparing different DNA isolation methods. J. Vet. Med. B. 53:281-285.

(NCCLS) National Committee for Clinical Laboratory Standards (1999). Performance Standards for Antimicrobial Susceptibility; Ninth Informational Supplement M100-S9. Villanova, PA, USA: NCCLS.

(NCCLS) National Committee for Clinical Laboratory Standards (2003). Approved standard M2-A8. Performance Standards for Antimicrobial Susceptibility. 8th ed. Wayne, PA.

(NCCLS) National Committee for Clinical Laboratory Standards (2000). Performance Standards for Antimicrobial Disk Susceptibility Tests. 4th Edn. Approved Standard M2-A7. NCCLS. Villanova Pa.

Olsen G, Woese C (1993). Ribosomal RNA: a key to phylogeny. FASEB. 7:113-123.

Paterson DL, Bonomo RA (2005). Extended-spectrum $\beta$-lactamases: a clinical update. Clin. Microbiol. Rev.18:657-686.

Paterson DL, Hujer KM, Hujer AM, Yeiser B, Bonomo MD, Rice LB, Bonomo RA (2003). Extended-spectrum B -lactamases in Klebsiella pneumoniae bloodstream isolates from seven countries: Dominance and widespread prevalence of SHV- and CTX-M-type $\beta$-lactamases. Antimicrob. Agents Chemother. 47:3554-3560.

Sanders CC, Peyret M, Moland ES, Cavalieri SJ, Shubert C, Thomson KS, Boeufgras JM, Sanders WE (2001). Potential impact on VITEK 2 system and Advanced Expert System on the clinical laboratory of a university-based hospital. J. Clin. Microbiol. 39:2379-2385.

Shah K, Mulla SA (2012). Susceptibility of ESBL-producing Enterobacteriaceae to ertapenem, meropenem and piperacillintazobactam. Nati.J. of Med. Res. 2(2):223-225.

Tang YW, Von Graevenitz A, Waddington MG, Hopkins MK, Smith DH, Li H, Kolbert CP, Montgomery SO, Persing DH (2000). Identification of coryneform bacterial isolates by ribosomal DNA sequence analysis. J. Clin. Microbiol. 38:1676-1678. 
Tenover FC (2007). Rapid detection and identification of bacterial pathogens using novel molecular technologies: infection control and beyond. Clin. Infect. Dis. 44: 418-423.

Tenover FC, Areibt RD, Goering RV, Mickelsen PA, Murray BE, Persing DH, Swaminathan B (1995). Interpreting chromosomal DNA restriction patterns produced by pulsed-field gel electrophoresis. J. Clin. Microbiol. 33: 2233-2239.

Weisburg WG, Barns SM, Pelletier DA, Lane DJ (1991). 16S ribosomal DNA amplification for phylogenetic study. J. Bacteriol. 173(2):697703.
Winokur PL, Canton R, Casellas JM, Legakis N (2001). Variations in the prevalence of strains expressing an extended-spectrum betalactamase phenotype and characterization of isolates from Europe, the Americas, and the Western Pacific region. Clin. Infect. Dis. 32: S94-S103.

Woodford N, Sundsfjord A (2005). Molecular detection of antibiotic resistance: when and where? J. Antimicrob. Chemother. 56:259-261. 